\title{
Effect of different levels of fish oil and canola oil on DMI, milk production and blood metabolites of high producing Iranian Holstein dairy cows in early lactation
}

T Vafa, A Naserian, A Heravi Moussavi, R Valizadeh, M Danesh Mesgaran

Ferdowsi University of Mashhad, Department of Animal Science, Mashhad, Khorasan, Islamic Republic of Iran

Email:vafa_toktam@yahoo.com

Introduction Supplemental fat sources are utilized in rations for dairy cows as a common method to increase the energy density of the diet to support energy demands for milk synthesis in early lactation (Heravi Moussavi et al, 2007). Although dietary fat supplementation increases the energy density of the diet, data on the relationship between the addition of dietary fat and the performance of lactating dairy cows are inconsistent because the impact of dietary fat is influenced by source of fat, method of processing, amount of fat included in the diet and stage of lactation. Canola seed which contains $40 \%$ fat is an excellent source of dietary fat high in unsaturated fatty acids and protein for dairy animals. Canola oil contains $51 \%$ oleic, 25\% linoleic, and 14\% linolenic acids. Fish oil (FO) also contains relatively high concentrations of two polyunsaturated fatty acids of the n-3 family: (EPA, C20:5) and (DHA, C22:6). Combination of fish oil with different plant oils has been studied previously but there are few studies which have examined the inclusion of fish oil in combination with canola oil. The aim of the present study, therefore, was to study the effects of different levels of fish oil and canola oil in early lactating Holstein cows.

Materials and mthods Eight multiparous early lactation Holstein cows (42 \pm 12 DIM, $40 \pm 6$ kg daily milk yield) were fed a total mixed ration supplemented with either $0 \%$ oil (Control), $2 \%$ canola oil (CO), $2 \%$ fish oil (FO), or $1 \%$ canola oil $+1 \%$ fish oil $(\mathrm{CoFo})$, according to a double $4 \times 4$ Latin square design. Each period lasted 3 wk; experimental analyses were restricted to the last week of each period. Diets were formulated according to NRC 2001. Cows were housed in tie stalls and fed the TMR two times a day to allow 5 to $10 \%$ orts (as-fed basis), and dry matter intake (DMI) was recorded at the last week of each period. Cows were milked 3 times per day and yields were recorded at each milking. Blood samples were taken from the coccygeal vein in the last day of each period, kept on ice and centrifuged within $30 \mathrm{~min}$ at $3000 \mathrm{x} \mathrm{g}$ for 20 min. Aliquots of serum were stored at $-20^{\circ} \mathrm{C}$ until analysis for glucose, insulin, triglyceride, cholesterol, and serum urea nitrogen (SUN). Data were analyzed as a replicated $4 \times 4$ Latin square using the GLM procedure of SAS (2001). The model included effects of diet, period and cow. Least squares means are reported throughout and significance was declared at $\mathrm{P}<0.05$.

Results The results of the effects of different levels of fish oil and canola oil are present on table 1 . There was no effect of $\operatorname{diet}(\mathrm{P}>0.05)$ on DMI, milk production, blood concentrations of glucose, urea, cholesterol, triglycerides or insulin.

Table 1 Influence of different levels of fish oil and canola oil in the diet on feed intake, milk yield and plasma metabolite concentrations.

\begin{tabular}{lllllll}
\hline \hline Parameter & \multicolumn{2}{l}{ Treatments } & & \multirow{2}{*}{ SEM } & \multirow{2}{*}{ p } \\
\cline { 2 - 5 } & Control & FO & FoCo & Co & & \\
\hline DMI, kg/d & 24.92 & 22.05 & 24.33 & 24.17 & 0.86 & 0.10 \\
Milk, kg/d & 34.41 & 32.93 & 34.55 & 34.15 & 0.84 & 0.62 \\
Glucose,mg/dl & 63.75 & 60.62 & 61.87 & 63.75 & 2.22 & 0.70 \\
Cholesterol,mg/dl & 192.87 & 211.75 & 207.5 & 194.37 & 8.75 & 0.40 \\
Triglyceride,mg/dl & 9.57 & 10.19 & 10.12 & 11.38 & 0.99 & 0.67 \\
Insulin, miclu/ml & 18.6 & 13.65 & 13.66 & 13.36 & 4.96 & 0.71 \\
SUN,mg /dL & 18.87 & 17.62 & 18.00 & 18.12 & 0.73 & 0.68 \\
\hline \hline
\end{tabular}

Conclusion Dietary inclusion of canola oil and fish oil had no effect on DMI, milk production or any blood metabolite measured. Withlock et al. (2002) reported similar results when fish oil and soybean oil were fed separately and in combination. Blood metabolite and insulin concentrations were also not different between diets consistent with previous studies (Khorasani and Kennelly, 1998).

\section{References}

Heravi, M.A.R., R.O. Gilbert, T.R. Overton, D.E. Bauman and W.R. Butler, 2007a. Effects of feeding fish meal and n-3 fatty acids on milk yield and metabolic responses in early lactating dairy cows. Journal of Dairy Science 90, $136-144$.

Khorasani, G.R., and J. J. Kennelly. 1998. Effect of Added Dietary Fat on Performance, Rumen Characteristics, and Plasma Metabolites of Midlactation Dairy Cows. Journal of Dairy Science 81,2459-2468.

Withlock, L.A., D. J. Schingoethe, A. R. Hippen, K. F. Kalscheur, R. J. Baer, N. Ramaswamy, and K. M. Kasperson. 2002. Fish oil and extruded soybeans fed in combination increase conjugated linoleic acids in milk of dairy cows more than when fed separately. Journal of Dairy Science. 85, 234-243. 